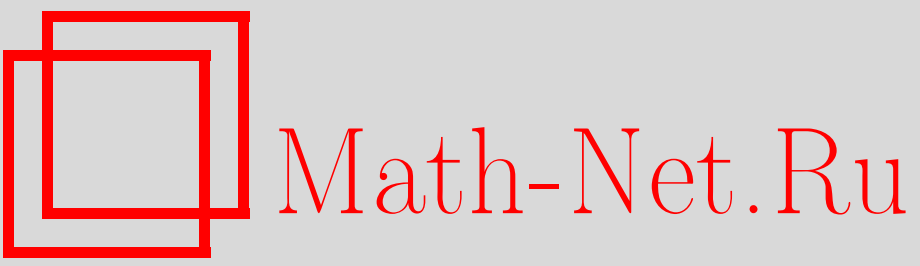

В. В. Зудилин, Совершенно уравновешенные гипергеометрические ряды и кратные интегралы, УМН, 2002, том 57, выпуск 4, 177-178

DOI: https://doi.org/10.4213/rm542

Использование Общероссийского математического портала Math-Net.Ru подразумевает, что вы прочитали и согласны с пользовательским соглашением

http://www . mathnet.ru/rus/agreement

Параметры загрузки:

IP: 3.81 .55 .215

26 апреля 2023 г., 15:01:44 


\title{
СОВЕРШЕННО УРАВНОВЕШЕННЫЕ ГИПЕРГЕОМЕТРИЧЕСКИЕ РЯДЫ И КРАТНЫЕ ИНТЕГРАЛЫ
}

\author{
В.В. Зудилин
}

Цель данной заметки - установить связь между двумя объектами: совершенно уравновешенными гипергеометрическими рядами

$$
\begin{aligned}
& F_{k}(\boldsymbol{h})=F_{k}\left(h_{0} ; h_{1}, \ldots, h_{k}\right):=\sum_{\mu=0}^{\infty}\left(h_{0}+2 \mu\right) \frac{\prod_{j=0}^{k} \Gamma\left(h_{j}+\mu\right)}{\prod_{j=0}^{k} \Gamma\left(1+h_{0}-h_{j}+\mu\right)}(-1)^{(k+1) \mu}
\end{aligned}
$$

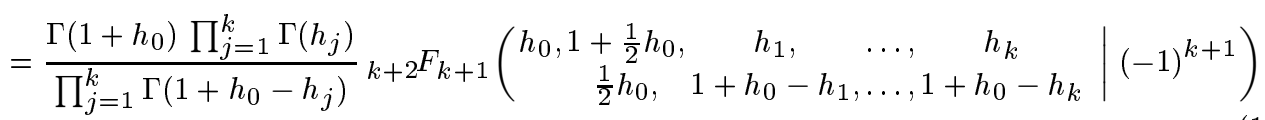

и кратными интегралами

$$
\begin{aligned}
J_{k}(\boldsymbol{a}, \boldsymbol{b}) & =J_{k}\left(\begin{array}{r}
a_{0}, a_{1}, \ldots, a_{k} \\
b_{1}, \ldots, b_{k}
\end{array}\right) \\
& :=\int_{[0,1]^{k}} \ldots \int_{0} \frac{\prod_{j=1}^{k} x_{j}^{a_{j}-1}\left(1-x_{j}\right)^{b_{j}-a_{j}-1}}{\left(1-\left(1-\left(\cdots\left(1-\left(1-x_{k}\right) x_{k-1}\right) \cdots\right) x_{2}\right) x_{1}\right)^{a_{0}}} \mathrm{~d} x_{1} \mathrm{~d} x_{2} \cdots \mathrm{d} x_{k} .
\end{aligned}
$$

ТеОрема. Пусть $k \geqslant 1$ и параметры $h_{0}, h_{1}, \ldots, h_{k+2} \in \mathbb{C}$ удовлетворяют условиям

$$
1+\operatorname{Re} h_{0}>\frac{2}{k+1} \cdot \sum_{j=1}^{k+2} \operatorname{Re} h_{j}, \quad \operatorname{Re}\left(1+h_{0}-h_{j+1}\right)>\operatorname{Re} h_{j}>0 \quad \text { для } j=2, \ldots, k+1
$$

и $h_{1}, h_{k+2} \neq 0,-1,-2, \ldots$. Тогда имеет место тождество

$$
\begin{aligned}
& \frac{\prod_{j=1}^{k+1} \Gamma\left(1+h_{0}-h_{j}-h_{j+1}\right)}{\Gamma\left(h_{1}\right) \Gamma\left(h_{k+2}\right)} \cdot F_{k+2}\left(h_{0} ; h_{1}, \ldots, h_{k+2}\right) \\
& \quad=J_{k}\left(\begin{array}{ccc}
h_{1}, \quad h_{2}, & h_{3}, \quad \ldots, & h_{k+1} \\
1+h_{0}-h_{3}, 1+h_{0}-h_{4}, \ldots, 1+h_{0}-h_{k+2}
\end{array}\right)
\end{aligned}
$$

Доказательство проводится методом математической индукции. Для $k=1$ утверждение теоремы следует из предельного случая теоремы Дугалла $[1, \S 4.4$, формула (1)]. Для $k \geqslant 2$, полагая $\varepsilon_{k}=0$ при $k$ четном и $\varepsilon_{k}=1$ или -1 при $k$ нечетном, мы пользуемся соотношением

$$
\begin{aligned}
J_{k}\left(\begin{array}{r}
a_{0}, a_{1}, \ldots, a_{k-1}, a_{k} \\
b_{1}, \ldots, b_{k-1}, b_{k}
\end{array}\right)= & \frac{\Gamma\left(b_{k}-a_{k}\right)}{\Gamma\left(a_{0}\right)} \cdot \frac{1}{2 \pi i} \int_{-t_{0}-i \infty}^{-t_{0}+i \infty} \frac{\Gamma\left(a_{0}+t\right) \Gamma\left(a_{k}+t\right) \Gamma(-t)}{\Gamma\left(b_{k}+t\right)} e^{\varepsilon_{k} \pi i t} \\
& \times J_{k-1}\left(\begin{array}{r}
a_{0}+t, a_{1}+t, \ldots, a_{k-1}+t \\
b_{1}+t, \ldots, b_{k-1}+t
\end{array}\right) \mathrm{d} t
\end{aligned}
$$

где $t_{0} \in \mathbb{R}, \operatorname{Re} a_{0}>t_{0}>0, \operatorname{Re} a_{k}>t_{0}>0, \operatorname{Re} b_{k}>\operatorname{Re} a_{0}+\operatorname{Re} a_{k}$ и интеграл в левой части (4) сходится. Представляя гипергеометрический ряд (1) в виде контурного интеграла Барнса и применяя к подынтегральному выражению в правой части (4) индукционное предположение, мы получаем требуемое тождество (3).

Отметим, что ряд в правой части (3) допускает 'менее экономичное' представление в виде эйлерова кратного интеграла по кубу $[0,1]^{k+2}$ (см. [2, лемма 1]). Из приведенной теоремњ и недавних результатов С.А. Злобина [3], [4] вытекает также представление совершенно уравновешенного гипергеометрического ряда (1) в виде кратного интеграла, предложенного в работах В.Н. Сорокина [5], [6]. 
Несмотря на аналитический характер теоремш, тождество (3) мотивировано арифметическими резултатами для значений дзета-функции Римана (дзета-значений) в целых положительных точках [5]-[13]. Известно [13], что в случае целочисленных параметров $\boldsymbol{h}$ совершенно уравновешенный гипергеометрический ряд (1) является $\mathbb{Q}$-линейной формой от четных или нечетных дзета-значений в зависимости от четности $k \geqslant 4$. Поэтому если целье положительные параметры $\boldsymbol{a}, \boldsymbol{b}$ удовлетворяют дополнительному условию

$$
b_{1}+a_{2}=b_{2}+a_{3}=\cdots=b_{k-1}+a_{k},
$$

то интеграл $(2)$ является $\mathbb{Q}$-линейной формой от дзета-значений одинаковой четности. Специализация $a_{j}=n+1, b_{j}=2 n+2$ приводит к совпадению кратных интегралов и совершенно уравновешенных гипергеометрических рядов, высказанному нами в качестве гипотезы в [13, 99$]$; обозначая соответствующие интегралы (2) через $J_{k, n}$ и применяя ариффметические результаты из [12, леммы 4.2-4.4], мы заключаем, что

$$
D_{n}^{k+1} \Phi_{n}^{-1} \cdot J_{k, n} \in \mathbb{Z} \zeta(k)+\mathbb{Z} \zeta(k-2)+\cdots+\mathbb{Z} \zeta(3)+\mathbb{Z} \text { для нечетного } k,
$$

где $D_{n}$ - наименьшее общее кратное чисел $1,2, \ldots, n$, а $\Phi_{n}$ - произведение простых чисел $p<n$, для которых $2 / 3 \leqslant\{n / p\}<1\left(\{\cdot\}\right.$ - дробная часть числа). Включения (6) (с множителем $D_{n}^{k}$ вместо $D_{n}^{k+1} \Phi_{n}^{-1}$ ) были предположены Д. Васильевым [14] (см. также [11, комментарий к теореме 2]) и доказаны им для $k=5$ (случай $k=3$ разобран в [7]). Таким образом, мы даем частичньй ответ к гипотезе Васильева. Выбор $a_{j}=r n+1, b_{j}=(r+1) n+2$ в (2) (или, эквивалентно, $h_{0}=(2 r+1) n+2$ и $h_{j}=r n+1$ для $j=1, \ldots, k+2$ в (1)) с цельм $r \geqslant 1$, зависящим от заданного нечетного $k$, приводит к почти тем же линейным формам от нечетных дзета-значений, рассмотренным Т. Ривоалем [10] в доказательстве его замечательного резултата о бесконечности множества иррациональных чисел среди $\zeta(3), \zeta(5), \zeta(7), \ldots$

Кроме того, следует отметить очевидную инвариантность в предположении (5) величины

$$
\frac{F_{k+2}\left(h_{0} ; h_{1}, \ldots, h_{k+2}\right)}{\prod_{j=1}^{k+2} \Gamma\left(h_{j}\right)}=\frac{J_{k}(\boldsymbol{a}, \boldsymbol{b})}{\prod_{j=1}^{k} \Gamma\left(a_{j}\right) \cdot \Gamma\left(b_{1}+a_{2}-a_{0}-a_{1}\right) \cdot \prod_{j=1}^{k} \Gamma\left(b_{j}-a_{j}\right)}
$$

под действием ( $\boldsymbol{h}$-тривиальнй) группы $\mathfrak{G}$ порядка $(k+2)$ !, состоящей из всех перестановок параметров $h_{1}, \ldots, h_{k+2}$. Этот результат также имеет теоретико-числовые приложения. В случаях $k=2$ и $k=3$ замена переменных $\left(x_{k-1}, x_{k}\right) \mapsto\left(1-x_{k}, 1-x_{k-1}\right)$ в $(2)$ реализует дополнительное преобразование с как интеграла (2), так и ряда (1); для $k \geqslant 4$ это преобразование недоступно, поскольку нарушается условие (5). Группы $\langle\mathfrak{G}, \mathfrak{c}\rangle$ порядков 120 и 1920 для $k=2$ и $k=3$ соответственно известны [8], [9]; Дж. Рин и К. Виола применяют их, чтобы получить хорошие оценки мер иррациональности чисел $\zeta(2)$ и $\zeta(3)$. В случае $k \geqslant 4$ группа $\mathfrak{G}$ допускает естественную интерпретацию как группа перестановок параметров $e_{0 l}=h_{l}-1,1 \leqslant l \leqslant k+2$, и $e_{j l}=h_{0}-h_{j}-h_{l}, 1 \leqslant j<l \leqslant k+2$ (детали см. в $\left.[13, \S 9]\right)$.

\section{СПИСОК ЛИТЕРАТУРЫ}

[1] W. N. Bailey. Generalized hypergeometric series. New York: Stechert-Hafner, 1964. (Cambridge Math. Tracts. V. 32.) [2] T. Rivoal, W. Zudilin // Prépubl. de l'Institut de Math. de Jussieu № 315 (janvier 2002). Paris, 2002. [3] С. А. Злобин // Матем. заметки. 2002. Т. 71 . № 5. С. 782-787. [4] С. А. Злобин // УМН. 2002. Т. 57. № 3. С. 153-154. [5] В. Н. Сорокин // Матем. сб. 1996. Т. 187. № 12. С. 87-120. [6] В. Н. Сорокин // Вестн. МГУ. Сер. 1. Матем., мex. 1998. № 3. C. 48-52. [7] F. Beukers // Bull. London Math. Soc. 1979. V. 11. № 3. P. 268-272. [8] G. Rhin, C. Viola // Acta Arith. 1996. V. 77.№ 1. P. 23-56. [9] G. Rhin, C. Viola // Acta Arith. 2001. V. 97. № 3. P. 269-293. [10] T. Rivoal // C. R. Acad. Sci. Paris Sér. I Math. 2000. V. 331. № 4. Р. 267-270. [11] Д. В. Васильев // Докл. НАН Беларуси. 2001. Т. 45. № 5. С. 36-40. [12] В. В. Зудилин // Изв. РАН. Сер. матем. 2002. Т. 66. № 3. С. 49-102. [13] W. Zudilin // E-print math. NT/0206176 (August 2001). [14] D. V. Vasilyev // Preprint no. 1(558). Minsk: Nat. Acad. Sci. Belarus, Institute Math., 2001.

Московский государственньй университет им. М. В. Ломоносова E-mail: wadim@ips.ras.ru
Принято редколлегией 03.06.2002 